\title{
Whitening efficacy of plant extracts including Hippophae rhamnoides and Cassia fistula extracts on the skin of Asian patients with melasma
}

\author{
Barkat Ali Khan ${ }^{1}$, Naveed Akhtar', Irshad Hussain ${ }^{1}$, Khwaja Asad Abbas², Akhtar Rasul $^{3}$ \\ 1Department of Pharmacy, Faculty of Pharmacy and Alternative Medicine, The Islamia University of Bahawalpur, Pakistan \\ 2Department of Botany, Government Sadiq Edgerton College, Bahawalpur, Pakistan \\ ${ }^{3}$ Faculty of Pharmacy, University of Sargodha, Pakistan
}

Postep Derm Alergol 2013; XXX, 4: 226-232

DOI: 10.5114/pdia.2013.37032

\begin{abstract}
Introduction: Melasma/hyperpigmentation and solar damage of the skin remains a difficult problem to treat. Various types of whitening agents are used to treat hyperpigmentation. A change has been observed recently to use plant extracts as skin whitening agents.

Aim: To compare the effectiveness of emulsion formulations containing plant extracts that include catechins/polyphenols and placebo without plant extracts, on patients with melasma.

Material and methods: Two groups of 25 patients each (aged 21-35 years), who reported to the outpatient department of BV Hospital and Personal clinic of a dermatologist, were included in the study. Volunteers applied the formulations with plant extracts and placebo to one side of the cheek. Prior to the study, signed consent was obtained from each patient. The tyrosinase inhibitory activity of the extracts and formulations was tested in vitro. The pigment density of patients was evaluated biometrologically using Mexameter ${ }^{\circledR}$ and subjectively using a visual survey before and after treatment of 12 weeks. The approval of the Institutional Ethics Committee of Faculty of Pharmacy, the Islamia University of Bahawalpur was obtained before the study. One-way ANOVA and Kruskal-Wallis tests were used in the statistical analysis.

Results: A significant decrease in the level of melanin was determined in all 50 patients who used a plant extract containing catechin $(p \leq 0.05)$. The difference between pre- and post-treatment levels of melanin was statistically significant $(p=0.05)$. Formulations prepared with plant extracts containing catechin were found effective on melasma, compared to the placebo.

Conclusions: Formulations containing plant extracts that are not yet being used widespread commercially on melasma could be an effective alternative treatment of melasma.
\end{abstract}

Key words: mexameter, melasma, Hippophae rhamnoides, Cassia fistula.

\section{Introduction}

Melasma is a general disorder of hyperpigmentation and usually involves the face and neck. Hyperpigmentation is particularly common in darker complected patients and is often hard to treat [1]. Skin-lightening agents are used to treat hyperpigmentation. Researchers have discovered and conducted various in vivo and in vitro studies on skinlightening agents. Arbutin, hydroquinone and several plant extracts are tried to achieve the skin-lightening effect and are being used in cosmetics. Skin-lightening agents inhibit the conversion of tyrosine to melanin by the enzyme tyrosinase, thus tyrosinase is the rate-limiting enzyme in melanin synthesis. Tyrosinase is a copper-containing enzyme that catalyzes melanin biosynthesis in three steps: the hydroxylation of tyrosine to 3,4-dihydroxyphenylalanine (DOPA), oxidation of DOPA to DOPA quinone and oxidation of 5,6-dihydroxyindole to indolequinone. Tyrosinase inhibitors play a vital role as skin-lightening agents [2].

Address for correspondence: Barkat Ali Khan PhD, Department of Pharmacy, Faculty of Pharmacy and Alternative Medicine, The Islamia University of Bahawalpur, Bahawalpur 63100, Pakistan, phone: +923339732578, fax: +92629255243, e-mail: barki.gold@gmail.com Received: 30.03.2013, accepted: 23.05.2013. 
Many plant extracts have a good inhibitory effect on melanin formation and may be a good choice for cosmetic purposes of whitening facial skin and defense against skin darkening. In addition, they have comparatively less side effects [3]. In cosmetic preparations, many plant extracts such as Morus alba, Acacia bark, Glycyrrhiza glabra and Green tea have been used as whitening agents [4-7].

Cassia fistula L, also known as the Golden Shower, is widely used in various countries as an ornamental tree for its beautiful bunches of yellow flowers. Recognized by the British Pharmacopoeia [8], C. fistula, a member of the Leguminosae family, is extensively used for its medicinal properties. In the Indian literature, this plant has also been described to be useful in skin diseases $[9,10]$. Cassia fistula is a medicinal plant acknowledged to be rich in phenolics, containing catechin, epicatechin, Kaempferol, ellagic acid (EA) and Rhein [11].

The medicinal value of Hippophae rhamnoides was found more than 1000 years ago, but it has been used for making medicines and healthy products by using modern science and technology only for the last 30 years [12]. Various catechin like (+)-gallocatechin and (-)-epigallocatechin have been isolated from $H$. rhamnoides berries [13].

\section{Aim}

The aim of this study was to investigate the effects of formulations containing phenolic-rich (catechin) extract of C. fistula and H. rhamnoides in vitro and in vivo.

\section{Material and methods}

\section{Plant materials and preparation of plant extracts}

Cassia fistula pods were collected from the Islamia University of Bahawalpur in August. Hippophae rhamnoides berries were purchased from Pak Sea Buckthorn International Skardu, Pakistan in May. The identification of the plant materials was performed by a taxonomist, Prof. Dr. Muhammad Arshad at the Cholistan Institute of Desert Studies (CIDS), the Islamia University of Bahawalpur and voucher specimens were preserved (voucher \# OB-LF-4-11-21 and HR-FT-03-11-23) at the herbarium of the Faculty of Pharmacy, Islamia University of Bahawalpur.

Plant materials were air-dried and then powdered. Each powdered plant was extracted with $70 \%$ methanol solution, and after filtration, the filtrates were evaporated under vacuum. These extracts were used for further studies of tyrosinase inhibition and emulsion formulations.

\section{Drugs and chemicals}

Methanol, catechin and gallic acid were obtained from Sigma (St Louis, MO, USA). Paraffin oil, Stearic acid, Tween80, Span20, Bees wax and Cetomacrogol were taken from Merck (Germany).

\section{In-vitro tyrosinase inhibitory activity of plant extracts}

Tyrosinase inhibitory activity is usually determined using a spectrophotometer. The procedure is briefly described here: a reaction mixture of $100 \mu \mathrm{l}, 40 \mu \mathrm{l} 100 \mathrm{mM}$ phosphate buffer, $\mathrm{pH}$ 6.8, $20 \mu \mathrm{l}$ mushroom tyrosinase enzyme (6 units) and $10 \mu \mathrm{l} 0.5 \mathrm{mM}$ test compound mixed in 96-well plate. The mixture was pre-incubated at $37^{\circ} \mathrm{C}$ for $5 \mathrm{~min}$. Thirty $\mu$ l of $10 \mathrm{mM} \mathrm{L-dopamine} \mathrm{(substrate)} \mathrm{was} \mathrm{added.} \mathrm{Contents}$ were mixed and incubated for 10 min more. Absorbance was taken at $\lambda 490 \mathrm{~nm}$ using Synergy HT BioTek 96-well plate reader. The percent enzyme inhibition was calculated as: $\%$ Inhibition $=100-($ Abs of test sample/Abs of control $\times 100$ ).

We determined the effect of the test compound on tyrosinase inhibition by $\mathrm{IC}_{50}$ using EZ-Fit Enzyme Kinetics Software (Perrella Scientific Inc., Amherst, USA).

\section{Preparation of formulations}

For investigation of the tyrosinase inhibitory effect of formulations, an oil-in-water (O/W) type emulsion (F1/F2) containing plant extracts and a placebo (F3) without plant extracts were prepared. The composition of the formulations is given in Table 1. The formulations were found stable for $\mathrm{pH}$, centrifugation, electrical conductivity, temperature stability tests at $8 \pm 0.1^{\circ} \mathrm{C}, 25 \pm 0.1^{\circ} \mathrm{C}, 40 \pm 0.1^{\circ} \mathrm{C}$ and $40 \pm 0.1^{\circ} \mathrm{C}$ with $75 \%$ relative humidity, physical nature i.e. color, creaming and liquefaction and rheological characteristics.

\section{In-vitro tyrosinase inhibitory activity of formulations}

Ozer et al. method with a slight modification was applied for in-vitro tyrosinase inhibitory activity of formulations [14]. Approximately $1.5 \mathrm{gm}$ of F1, F2 and F3 was weighed accurately into a centrifuge tube. Ten $\mathrm{ml}$ of methanol (50\%) was added and stirred in an ultrasonic bath for $30 \mathrm{~min}$. The suspension was centrifuged at $5000 \mathrm{rpm}$ for $10 \mathrm{~min}$. It was further diluted at a ratio of $1: 10$ with $50 \%$ methanol and tested by the dopachrome method for determination of tyrosinase inhibitory effect.

Table 1. Composition of formulations (\% w/w)

\begin{tabular}{lcc}
\hline Composition & F1/F2 & F3 \\
\hline Liquid paraffin & 24 & 24 \\
\hline Stearic acid & 5 & 5 \\
\hline Span 20 & 2 & 2 \\
\hline Bees wax & 7 & 7 \\
\hline Cetomacrogol & 5 & 5 \\
\hline Tween 80 & 6 & 6 \\
\hline Plant extract & 5 & Nil \\
\hline Preservative & 1 & 1 \\
\hline D/W & 45 & 50 \\
\hline
\end{tabular}




\section{Subjects}

Two groups of 25 healthy subjects each (aged 21-35) were enrolled in this study after their informed consent was obtained. All volunteers completed the study successfully. None of them had any pathological condition on their cheeks. They were sufficiently informed about the use of products. Moreover, they were told not to use any other skin products, especially whitening agents, throughout the 12-week duration of the study.

\section{In-vivo study design}

A single blinded, placebo-controlled study was conducted to assess the effects of formulations on the improvement of the skin melanin (anti-melasma effects). This study was conducted in October-December and an expert investigator ensured the proper handling of biometrological measurements, allowing for obtaining uniform results. Tests were carried out on both the right and left cheeks of volunteers. Measurements were carried out at baseline and on week 1, 2, 3, 4, 6, 8, 10 and 12 .

Approximately $500 \mathrm{mg}$ of formulations were applied to the cheeks twice daily (mornings and evenings) over a period of 12 weeks. Prior to all measurements, volunteers were asked for at least 15 min wait in order to allow for complete skin adjustment to the room temperature.

\section{Ethical considerations}

The institutional ethics committee of the Faculty of Pharmacy, the Islamia University of Bahawalpur approved the protocol of this study (No. 942/Acad). Before enrollment and inclusion of a volunteer in this study, signed informed consent was obtained from each volunteer.

\section{Instrumental measurements}

Biometrologically, a Mexameter ${ }^{\circledR}$ (Courage \& Khazaka, Germany) with noninvasive probes was used in this study for assessing the skin melanin. Mexameter ${ }^{\circledR}$ is a narrow-band reflectance spectrophotometer, designed to measure the intensity of skin melanin. It has mainly been used for cosmetic research but recently it has also been used to assess hyperpigmentation of skin (melasma).

Table 2. Tyrosinase inhibition activity of H. rhamnoied and C. fistula extracts

\begin{tabular}{|c|c|c|}
\hline \multirow[t]{2}{*}{ Plant extracts } & \multicolumn{2}{|c|}{ Tyrosinase inhibition } \\
\hline & $\begin{array}{c}\text { Average inhibition } \\
\text { percentage (\%) at } 0.05 \mathrm{mg}\end{array}$ & $\mathrm{IC} 50[\mu \mathrm{g}]$ \\
\hline H. rhamnoides & $72.57 \pm 0.34^{a}$ & 45.9 \\
\hline C. fistula & $77.64 \pm 0.62^{b}$ & 39.2 \\
\hline Control & Kojic acid & $6.00 \pm 0.01$ \\
\hline
\end{tabular}

All values were based on three different samples. All assays were determined in triplicates per run and data presented as mean $\pm S D$. For column of each sample, values followed by the same letter are not significantly different at $p<0.05$

\section{Visual survey}

After 12 weeks of treatment, a visual survey was carried out to indicate 3 parameters: change in photograph, change in size and change in pigmentation. Photographs of only those volunteers, who allowed openly for reproduction, were taken.

\section{Mathematical analysis}

The percentage changes in melanin after every week for each volunteer were calculated by using the following formula: \%Change $=[(\mathrm{T} x-\mathrm{TO}) / \mathrm{TO}] \times 100$, where: T0 - baseline values, $\mathrm{Tx}$ - value obtained every week.

\section{Statistical analysis}

The measured values were analyzed using GraphPad Prism version 5 statistical software. A paired sample t-test for variation between the active formulation and placebo was performed. Standard Parametric one-way ANOVA with Dunnett Multiple Comparisons Post Test was used to observe the correlation between baseline values and the values at different weeks. Values of $p$ of less than 0.005 were considered statistically significant.

\section{Patch Test (Burchard Test)}

Prior to sampling, a patch test was performed to find any possible sensitivity of formulations on forearms of each volunteer. A specified area was marked on the forearms. Placebo was applied to the right forearm while the active formulation was applied to the left forearm. The areas were covered with surgical dressing (Patches) saturated with formulations. After $48 \mathrm{~h}$, the patches were removed and the forearms were washed with saline. Scores were noted for the presence of itching/irritation using a 4-point scale ranging from 0 to 3; where 3 stands for severe response, 2 for moderate, 1 for mild and 0 stands for no response. Each volunteer was requested to note the degree of itching/ irritation and allocate a score from the same scale.

\section{Results}

\section{In-vitro tyrosinase inhibitory activity of plant extracts and formulations}

Table 2 summarizes the results of anti-tyrosinase inhibition activity of plant extracts, where inhibition is expressed as $I C_{50}$ values. Results indicate that $H$. rhamnoides had slight poor antityrosinase activity (72\% inhibition at $0.05 \mathrm{mg} / \mathrm{ml}$ concentration) as compared to C. fistula extracts ( $77 \%$ inhibition at $0.05 \mathrm{mg} / \mathrm{ml}$ concentration). Evaluation of the formulations on tyrosinase inhibition confirmed that the active formulations (F1 and F2) containing plant extracts at 5\% concentration have the inhibitor activity. Placebo without any plant extract was used as a control sample, and no inhibitory effect was observed. Tyrosinase inhibitory effects of formulations are given in Table 3. 


\section{Patch test}

There was no severe response of itching/irritation in any volunteer, mild response for formulation F1 and F2 occurred in 4 volunteers each, moderate erythema occurred in 2 and 4 volunteers, whereas no itching/irritation occurred in 19 and 17 volunteers, respectively, as shown in Table 4.

\section{In-vivo tyrosinase inhibitory activity of formulations (mexametric evaluation)}

The percent changes for the skin melanin following the 12 weeks' treatment with F1 and F3 along with statistical analysis are shown in Figure 1. The percent changes for the skin melanin following the 12 weeks' treatment with F2 and F3 along with statistical analysis are shown in Figure 2.

In the case of F1 and F3, it was noted that F3 has shown a mean initial change in melanin (2.52\%) after the $1^{\text {st }}$ week and the effect remained on the increase after the $12^{\text {th }}$ week up to $5.8 \%$ compared to the baseline melanin values of the 25 volunteers.

In contrast to the F3 treatment effects, an excellent reduction in the skin melanin was noticed after treatment with F1, i.e. $-3.5 \%$ mean initial reduction in melanin after the $1^{\text {st }}$ week of treatment, $-7.0 \%$ reductions after 1 month, and $16.35 \%$ reductions in melanin at the end of the $12^{\text {th }}$ week compared to baseline values. When one-way analysis of vari-
Table 3. Tyrosinase inhibitory effect of formulations $(n=3)$

\begin{tabular}{ll}
\hline Formulation & Tyrosinase inhibitory effect [\%] \\
\hline F1 & $58.6 \pm 0.425$ \\
\hline F2 & $64.0 \pm 0.107$ \\
\hline F3 & 0
\end{tabular}

F1 - Formulation containing H. rhamnoides extract, $F 2$ - formulation containing C. fistula extract, F3 - placebo

ance, ANOVA (Kruskal-Wallis test) was applied, there was a high significant $(p<0.0001)$ difference between F1 and F3. When Dunn's multiple comparison tests were applied, there were insignificant $(p>0.05)$ differences between various time intervals.

F2 produced a noticeable reduction in melanin with an initial mean reduction of $-7.07 \%$ after the $1^{\text {st }}$ week and the effect was maintained until the $12^{\text {th }}$ week up to $-13.0 \%$, compared to the baseline skin melanin values of the 25 volunteers. When one-way analysis of variance, ANOVA (KruskalWallis test) was applied, there was a significant $(p<0.0001)$ difference between F2 and F3. When Dunn's multiple comparison tests were applied, there were insignificant $(p>0.05)$ differences between various time intervals except the $2^{\text {nd }}$ and $4^{\text {th }}$ week and the $1^{\text {st }}$ and $12^{\text {th }}$ week, where significant results were obtained.

Table 4. Score allocated by volunteers to formulations on the basis of itching/irritation

\begin{tabular}{lcccc}
\hline Formulation type & \multicolumn{4}{c}{ Score } \\
\cline { 2 - 4 } & 0 & 1 & 2 & 3 \\
\cline { 2 - 4 } & & No. of volunteers indicating itching/irritation & 0 \\
\hline F1 & 19 & 4 & 2 & 0 \\
\hline
\end{tabular}

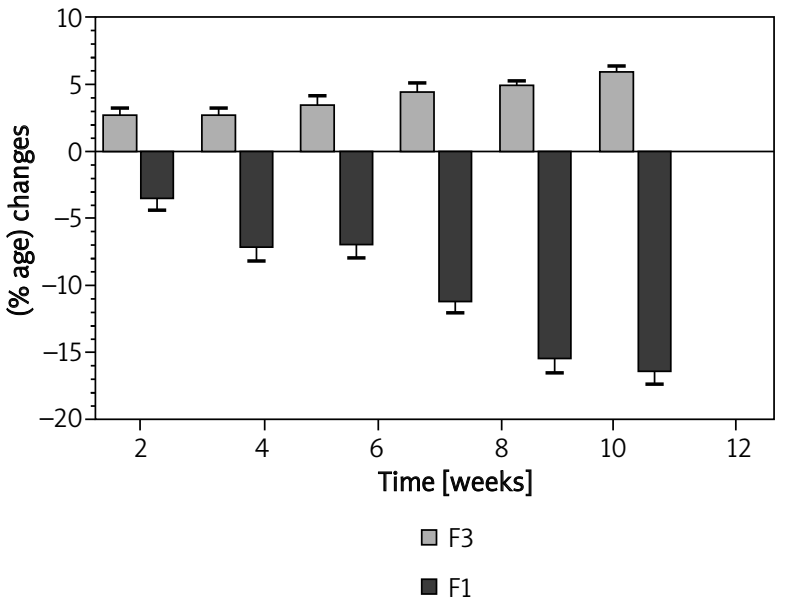

Fig. 1. Percentage of changes produced in skin melanin after the application of F3 (placebo) and F1 (H. rhamnoides)

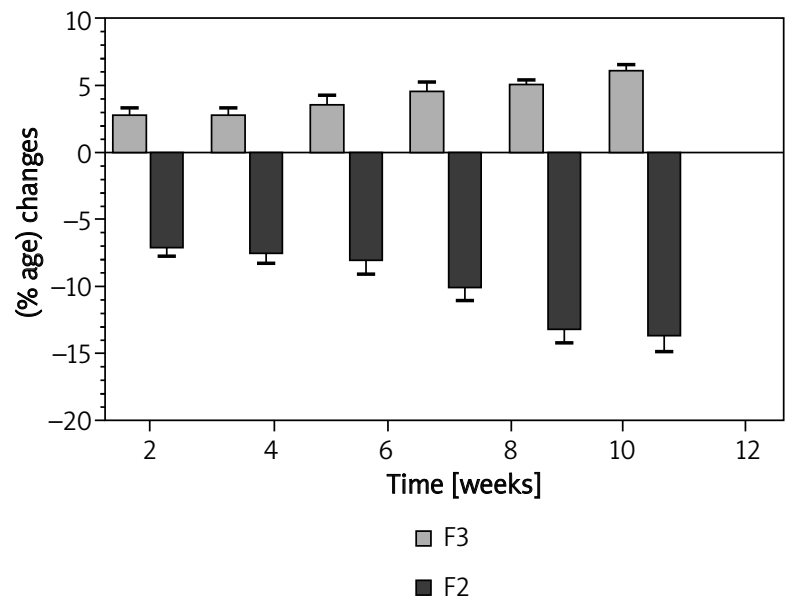

Fig. 2. Percentage of changes produced in skin melanin after the application of F3 (placebo) and F2 (C. fistula) 
Table 5. Visual survey of formulations

\begin{tabular}{|c|c|c|c|c|c|}
\hline Parameter & Condition & $\mathrm{F} 1(n=25)$ & $\mathrm{F} 2(n=25)$ & F3 $(n=25)$ & Value of $p$ \\
\hline \multirow{3}{*}{$\begin{array}{l}\text { Change } \\
\text { in photograph }\end{array}$} & Improved & 14 & 19 & 0 & 0.08 \\
\hline & Not improved & 10 & 4 & 44 & \\
\hline & Deteriorated & 1 & 2 & 6 & \\
\hline \multirow[t]{3}{*}{ Change in size } & Improved & 18 & 20 & 0 & 0.057 \\
\hline & Not improved & 5 & 4 & 40 & \\
\hline & Deteriorated & 2 & 1 & 10 & \\
\hline \multirow{3}{*}{$\begin{array}{l}\text { Change } \\
\text { in pigmentation }\end{array}$} & Improved & 18 & 19 & 0 & 0.055 \\
\hline & Not improved & 6 & 5 & 36 & \\
\hline & Deteriorated & 1 & 1 & 14 & \\
\hline \multirow[t]{3}{*}{ Net result } & Improved & 21 & 22 & 0 & 0.061 \\
\hline & Not improved & 3 & 2 & 42 & \\
\hline & Deteriorated & 2 & 1 & 8 & \\
\hline
\end{tabular}

\section{Visual survey}

Subjectively using a visual survey, the number of volunteers who responded to various parameters is shown in Table 5 for F1, F2, F3. In the paired sample $t$-test, an insignificant difference between the three conditions was observed, which showed that there was no variation between the active formulations.

\section{Discussion}

Hyperpigmentation is one of the most common skin disorders. An increase in melanin synthesis or an irregular dis- tribution of melanin results in hyperpigmentation or spots, especially on the face. Melasma is an example of pigmentation observed mainly in females, though in Asia it is commonly seen in males as well. It is more common in South Asian countries due to skin type IV to V (darker complexion) and increased UV radiation. Treating melasma is challenging and the main treating agent is hydroquinone. The major problem with high concentration (above $4 \%$ ) of hydroquinone is contact dermatitis. Though various other treatment options are also available, all are very costly with delayed results [15].

Various types of whitening agents are used to treat hyperpigmentation. These agents include arbutin, hydro-
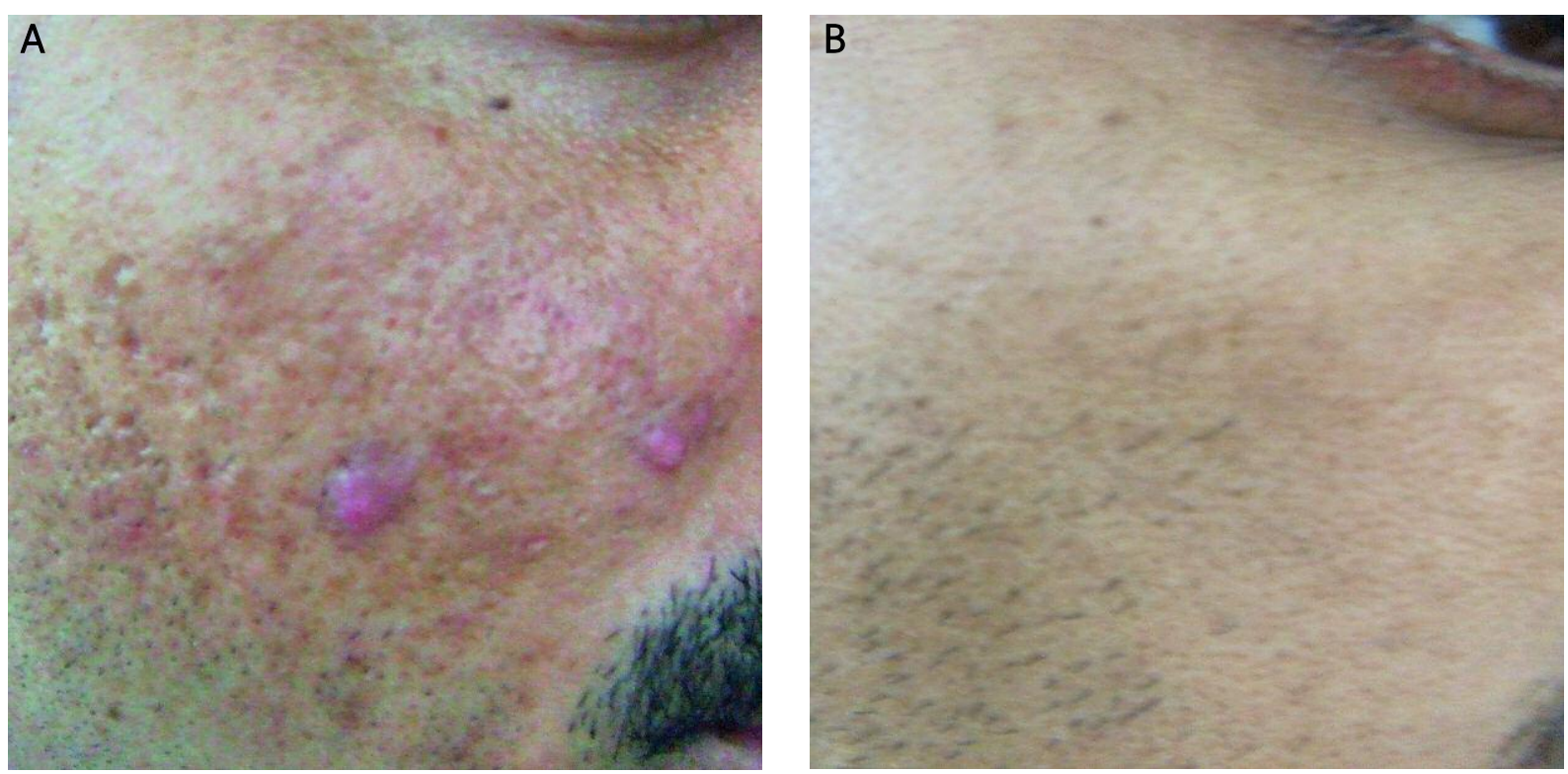

Fig. 3. A - Baseline photo of a patient treated with F1. B - Clinical improvement seen after 12 weeks of therapy with F1 

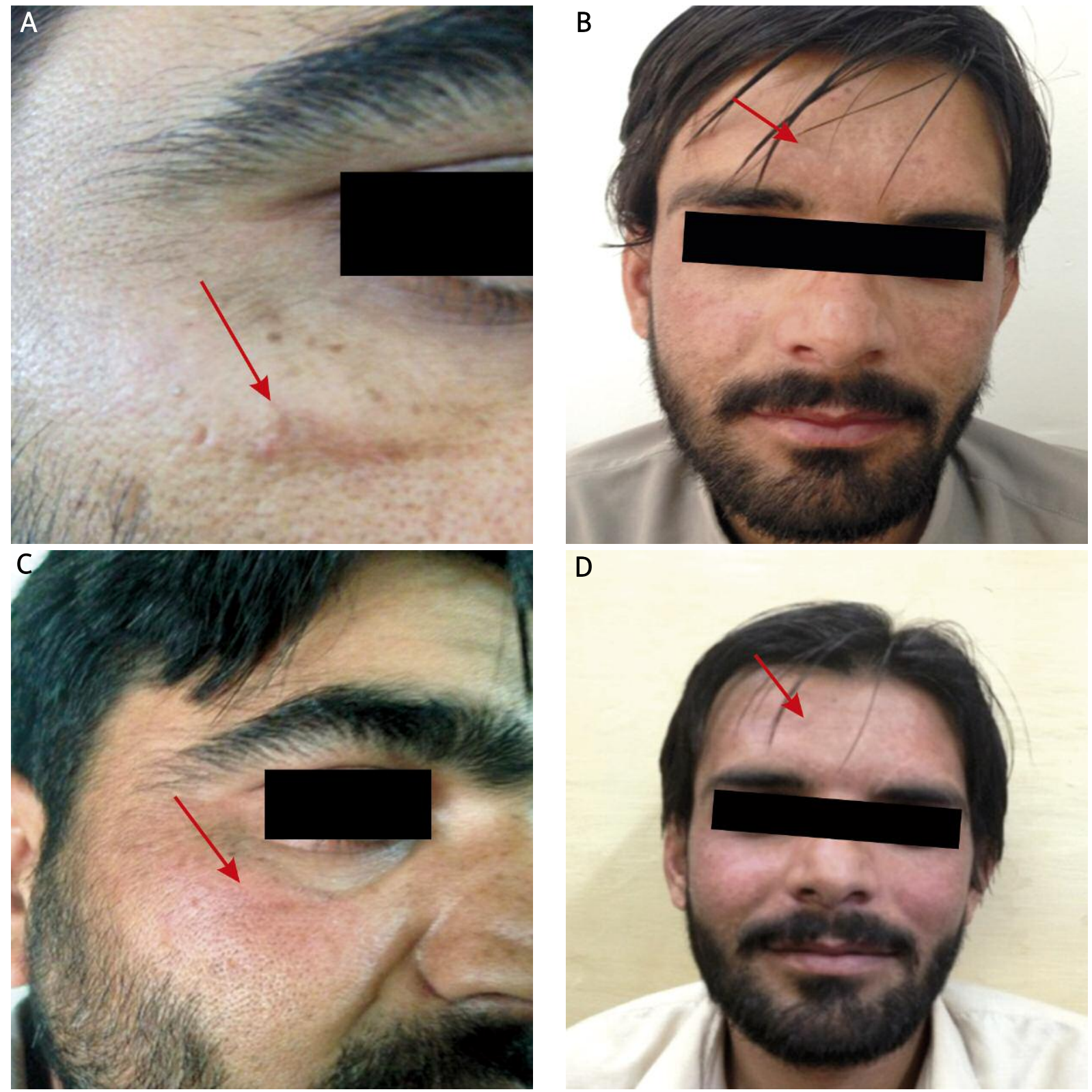

D

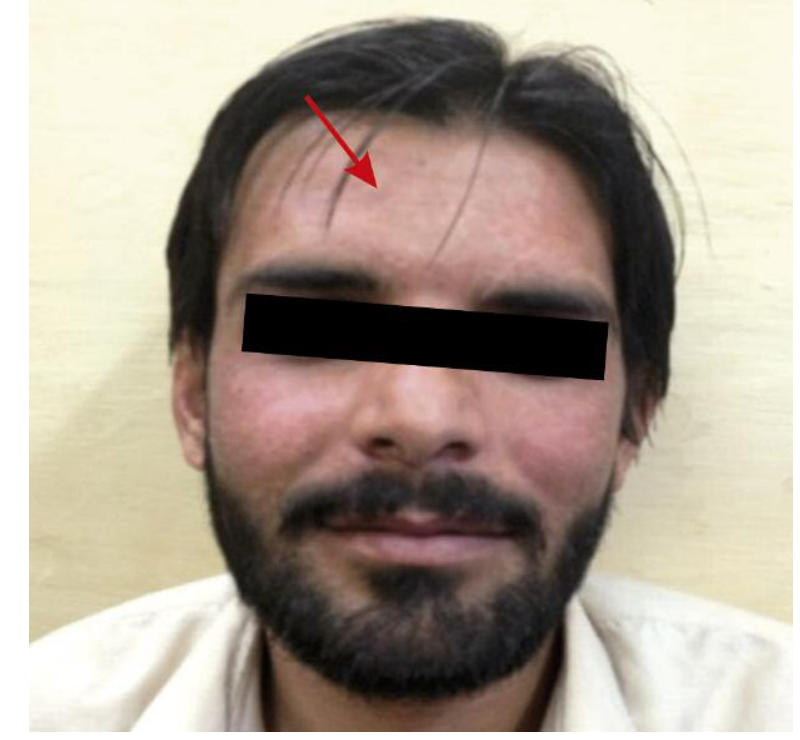

Fig. 4. A, B - Baseline photos of patients treated with F2. C, D - Clinical improvement seen after 12 weeks of therapy with F2

quinones, azelaic acid and kojic acid. Though these agents are effective, they have side effects. A change has been observed recently to use plant extracts as skin-whitening agents in different types of formulations [16].

It has been reported by several researchers that plants rich in alkaloids, flavonoids, unsaturated fatty acids (linoleic acid, oleic acid) and mineral have anti-tyrosinase activity $[17,18]$. Similarly, catechins, including (+)-gallocatechin, $(-)$ )-epicatechin, $(-)$ )-epigallocatechin (EGC), (-)) epicatechin gallate (ECG) and (-))-epigallocatechin gallate (EGCG) are studied for anti-tyrosinase activity [19].

Both plants, H. rhamnoides and C. fistula, are reported to contain catechins, gallic acid, flavonoids, quercetin, organic acids (linoleic acid, oleic acid). The reduction in skin melanin (anti-melasma effects) can be credited to these constituents present in F1 and F2.

\section{Conclusions}

The good agreement between the results of in-vitro/invivo evaluations and those of visual surveys showed that the plant extracts demonstrate efficacy in comparison to placebo in whitening the skin as well as melasma on the face of patients. The plant extracts, which demonstrated a significant tyrosinase inhibitory activity, were described for the first time to possess this biological property. In Pakistan, where sun exposure is severe, the pos- 
sibility of developing melasma is higher. Instead of expensive and risky treatments, there is a demand for natural remedies which are cheaper and effective. H. rhamnoides and $C$. fistula, therefore, offer a possible solution to the problem.

\section{Acknowledgments}

The authors thank the Higher Education Commission for financial support.

\section{References}

1. Torok HM. A comprehensive review of the long-term and short-term treatment of melasma with a triple combination cream. Am J Clin Dermatol 2006; 7: 223-30.

2. Kanthraj GR. Skin-lightening agents: New chemical and plant extracts-ongoing search for the holy grail! Indian J Dermatol Venereol Leprol 2010; 76: 3-6.

3. Kim J, Lee KT. Inhibitory effects of Ramulus mori extracts on melanogenesis. Cosmet Toilet 1998; 113: 65-70.

4. Lee KT, Kim BJ, Kim HJ, et al. Biological screening of 100 plant extracts for cosmetic use (I): inhibitory activities of tyrosinase and DOPA auto-oxidation. Int J Cosmet Sci 1997; 19: 291-8.

5. Bernard P, Berthon Y. Resveratrol: an original mechanism on tyrosinase inhibition. Int J Cosmet Sci 2000; 22: 219-26.

6. Baurin N, Arnoult E, Scior T, et al. Preliminary screening of some tropical plants for antityrosinase activity. J Ethnopharmacol 2002; 82: 155-8.

7. Ali A, Akhtar N, Shoaib Khan M. In vivo evaluation: the effects of a cream containing Acacia bark extract on skin melanin and erythema content. Postep Derm Alergol 2012; 29: 369-72.

8. Alam MM, Siddiqui MB, Hussian W. Treatment of diabetes through herbal drugs in rural India. Fitoter 1990; 61: 240-2.

9. Mukhopadhyay M, Saha A, Dutta A, et al. Genotoxicity of sennosides on the bone marrow cells of mice. Food Chem Toxicol 1998; 36: 937-40.

10. Asolkar LV, Kakkar KK, Chakre OJ. Second supplement to glossary of Indian medicinal plant with active principles. In: Publication and Information Directorate, New Delhi 1992; 177.

11. Kashiwada Y, Toshika K, Chen R, et al. Tannins and related compounds. XCIII. Occurrence of enantiomeric proanthocyanidins in the Leguminosae plants, Cassia fistula L.; Cassia Javanica L. Chem Pharm Bull 1996; 38: 888-93.

12. Yuzhen Z, Fuheng W. The flavonoids in Seabuckthorn and their medicinal value. Hippophae 1997; 10: 39-1.

13. Rösch D, Krumbein A, Kroh LW. Antioxidant gallocatechins, dimeric and trimeric proanthocyanidins from sea buckthorn (Hippophaë rhamnoides) pomace. Eur Food Res Technol 2004; 219: 605-13.

14. Ozer O, Mutlu B, Kivcak B. Antityrosinase activity of some plant extracts and formulations containing ellagic acid. Pharmaceut Biol 2007; 45: 519-24.

15. Shazia Z, Ghulam M. Comparison of efficacy of topical $2 \%$ liquiritin, topical $4 \%$ liquiritin and topical $4 \%$ hydroquinone in the management of melasma. J Pak Assoc Dermatol 2009; 19: 158-63.

16. Parvez S, Kang M, Chung H, et al. Survey and mechanism of skin depigmenting and lightening agents. Phytother Res 2006; 20: 921-34.
17. Mukherjee PK, Mukherjee D, Maji AK, et al. The sacred lotus (Nelumbo nucifera) - phytochemical and therapeutic profile. J Pharm Pharmacol 2009; 61: 407-22.

18. Ruksiriwanich W, Manosroi J, Abe M, et al. 5 alpha-reductase type 1 inhibition of Oryza sativa bran extract prepared by supercritical carbon dioxide fluid. I Supercrit Fluid 2011; 59: 61-71.

19. Ali A, Akhtar N, Khan MS. In vivo evaluation: the effects of a cream containing Acacia bark extract on skin melanin and erythema content. Postep Derm Alergol 2012; 29: 369-72. 